\title{
Adaptação do Algoritmo PSO para Problemas Multiobjetivo, Iterações com Liderança Global Fixa
}

\author{
Acir M. Soares Junior ${ }^{1}$ \\ UENF/LENEP - Macaé-RJ \\ Breno T. Mota ${ }^{2}$ \\ UERJ/Instituto Politécnico - Nova Friburgo-RJ \\ Luciana P. M. Pena ${ }^{3}$ \\ UFF/IME/GMA - Niterói-RJ \\ Adolfo P. Pires ${ }^{4}$ \\ UENF/LENEP - Macaé-RJ
}

\begin{abstract}
Resumo. A abordagem multiobjetivo (MO) associada a problemas de otimização tem estimulado o desenvolvimento e aperfeiçoamento de novos métodos, em especial os chamados algoritmos evolucionários. Dentre eles, o MOPSO tem recebido especial atenção devido, entre outros aspectos, ao número reduzido de parâmetros. Neste trabalho é apresentada uma nova versão do MOPSO, formulada a partir da estrutura original do PSO, acrescida de seis modificações, que tratam diferentes problemas resultantes da aplicação da teoria de Pareto aos algoritmos de otimização. A versão proposta foi testada comparativamente na solução de quatro problemas clássicos em relação a versão apresentada por Coello et al., 2004.
\end{abstract}

Palavras-chave. Algoritmo, MOPSO, multi objetivo, otimização.

\section{Introdução}

Os problemas de otimização multiobjetivo (MO) surgem nas mais diversas áreas como economia, administração, engenharia, robótica, telecomunicações, química e design industrial dentre outras, o que tem motivado a contínua investigação e o desenvolvimento de novos métodos e técnicas aplicados à solução destes problemas.

Sem a perda de generalidade, a otimização multiobjetivo pode ser definida como um problema de minimização do valor de uma função vetorial $\mathbf{F}$, que define a aplicação de um vetor com $n$ variáveis livres $\mathbf{X}$ em um vetor contendo $m$ objetivos $\mathbf{Y}$. As funções objetivo são em geral conflitantes, inexistindo um único vetor $\mathbf{X}$ que minimize todos os valores das funções objetivo $Y_{1}, Y_{2}, \ldots, Y_{m}$ simultaneamente.

\footnotetext{
${ }^{1}$ acir@lenep.uenf.br

${ }^{2}$ brenotsm1@gmail.com

${ }^{3}$ lucianapena@id.uff.br

${ }^{4}$ adolfo.puime@gmail.com
} 
A abordagem formal para este tipo de problema teve início com a teoria de Pareto em 1906, que estabeleceu critérios de otimalidade a partir do conceito de dominância de Pareto [2], onde a existência de múltiplos objetivos conflitantes resulta no surgimento de soluções múltiplas e equivalentes entre si.

Enquanto os métodos clássicos de otimização mono-objetivo tem como resultado de busca uma solução única, os algoritmos evolucionários podem encontrar múltiplas soluções em uma única rodada por serem baseados em meta-heurísticas populacionais. Diversos estudos tem demonstrado a utilidade e relevância do emprego de algoritmos evolucionários na solução de problemas multiobjetivos [9]. Dentre eles, o algoritmo MOPSO (do inglês "Multi Objective Particle Swarm Optimization"), construído a partir da sua versão mono objetivo (PSO) proposta por Kennedy e Eberhart em 1995, tornou-se muito popular devido a algumas características do algoritmo original como o reduzido número de parâmetros e capacidade de exploração global.

A adaptação do PSO para a resolução de problemas multiobjetivo (MOPSO) traz consigo uma série de desafios, que motivaram o desenvolvimento de diferentes abordagens e versões encontradas na literatura, onde a principal alteração decorre do surgimento de lideranças múltiplas. Nos problemas multiobjetivo clássicos, o ótimo global deixa de ser um único ponto e deve ser escolhido dentro de um conjunto de soluções que atendem a um critério de otimalidade, que se baseia no critério de dominância de Pareto [7].

Vários esquemas foram propostos para armazenar e manipular a seleção de líderes por meio de um arquivo externo [5], tornando-se fonte de estudo para aperfeiçoamento do método. Na versão projetada por Coello et al. (2004) [3] o espaço dos objetivos é divido em "hipercubos", desta forma um líder é escolhido por sorteio no cubo com menor número de partícula.

Neste trabalho, além da escolha, armazenagem e gerenciamento das lideranças múltiplas, também são abordadas outras três questões relevantes para a otimização multiobjetivo: o critério de dominância, a escolha do líder local entre posições equivalentes e o cálculo da velocidade das partículas.

\section{Esquema Numérico}

A versão do MOPSO proposta mantem a estrutura do PSO original [4], introduzindo seis modificações simplificadas ao algoritmo.

1. Critério de otimalidade: Em substituição à dominância de Pareto, adotou-se o critério de $\varepsilon$-dominância, implementado inicialmente por Laummans et al. [6] com o objetivo de promover uma melhor distribuição das partículas na imagem. A $\varepsilon$ dominância pode ser definida de maneira formal como: Considerando $F_{i}(\mathbf{X})=Y_{i}, i=$ $(1,2, \ldots, m)$ um conjunto de funções objetivo a serem minimizadas, então dados dois vetores $\mathbf{Y}^{*} \in \mathbb{R}^{m}$ e $\boldsymbol{\varepsilon} \in \mathbb{R}^{m}$, disse que $\mathbf{Y}^{*} \varepsilon$-domina $\mathbf{Y} \in \mathbb{R}^{m}$, denotado por $\mathbf{Y}^{*} \succ_{\varepsilon} \mathbf{Y}$ , se e somente se: $Y_{i}^{*}-\varepsilon_{i} \leq Y_{i} \forall i \in(1,2, \ldots, m)$. Nesta versão, o critério de $\varepsilon$ dominância foi implementado com o objetivo de eliminar problemas de dominância puramente numérica, que não oferecem um ganho real para o valor da função. 
2. Armazenagem da liderança global: o conjunto de partículas líder, isto é, o conjunto de partículas não dominadas, que corresponde a uma aproximação numérica da frente de pareto, é armazenado em um vetor de tamanho definido, denominado VFP, semelhante ao repositório externo utilizado por Coello e Lechuga [2].

3. Gerenciamento da armazenagem dos líderes globais: Quando o número de partículas não dominadas supera o número máximo de posições de armazenagem, elimina-se a partícula excedente, empregando-se o critério de menor distância euclidiana, operacionalizado por meio de uma matriz $\mathbf{D}$, que armazena as distâncias entre cada uma das partículas não dominadas. Assim, elimina-se aquela que apresenta os dois menores valores na matriz $\mathbf{D}$.

4. Escolha do líder global (gbest): A atualização da liderança para cada partícula do enxame é feita sem a utilização de quaisquer critérios de seleção. Os líderes globais, provenientes do vetor de partículas não dominadas (VFP) são atribuídos às partículas do enxame de forma sequencial a partir de um primeiro elemento escolhido aleatoriamente. Observou-se que a mudança constante do líder global pode gerar um movimento errático das partículas do enxame. Para contornar este problema e acelerar a convergência das partículas em direção à frente de Pareto, o líder global é mantido fixo por um determinado número de iterações, que varia entre 3 e 8 .

5. Cálculo da velocidade: A velocidade $(V)$ na iteração $k+1$ em cada uma das $i$ dimensões é calculada de acordo com a expressão original adicionada do fator de inércia $(\omega)$ sugerido por Chatterjee e Siarry [1] para aumentar a capacidade de exploração global no início e a capacidade de exploração local nas últimas iterações.

$$
V_{i}(k+1)=\omega \cdot V_{i}(k)+C_{1} \cdot \phi_{1}\left(X_{i} \text { pbest }(k)-X_{i}(k)+C_{2} \cdot \phi_{2}\left(X_{i} g \operatorname{best}(k)-X_{i}(k)\right)\right)
$$

onde o fator de inércia é calculado pela expressão $\omega=0,4+0,5\left(k_{\max }-k\right)^{1,2} /\left(k_{\max }\right)^{1,2}$; $\phi$ corresponde a um número aleatório entre 0 e $1 ; C_{1}=2,05$ corresponde ao fator de aprendizagem local; $C_{2}=2,05$ corresponde ao fator de aprendizagem global; $X_{\text {pbest }}$ e $X_{\text {gbest }}$ correspondem à posição da liderança local e global respectivamente.

6. Escolha da liderança local (pbest): A escolha é feita inicialmente pelo critério de $\varepsilon$-dominância. Caso as partículas sejam não dominantes entre si, é mantida a partícula cuja troca resultaria na maior perda relativa para uma das funções objetivo.

\section{Testes Comparativos}

Foram empregadas quatro funções testes, Eqs. (2) , (3) , (4) e (5), selecionadas por Coello et al. [3], e resolvidas com o emprego do MOPSO com um número máximo de avaliações da função objetivo de 5000, 12000, 4000 e 10000 para cada um dos testes respectivamente. Seguindo a mesma metodologia, cada problema foi resolvido 30 vezes com o algoritmo proposto, empregando os parâmetros indicados na Tabela 1. Os resultados foram comparados por meio da apresentação gráfica e das métricas GD e SP, do inglês "Generational Distance"e "Spacing"respectivamente [3]. 
Tabela 1: Parâmetros empregados nas resolução de cada função teste.

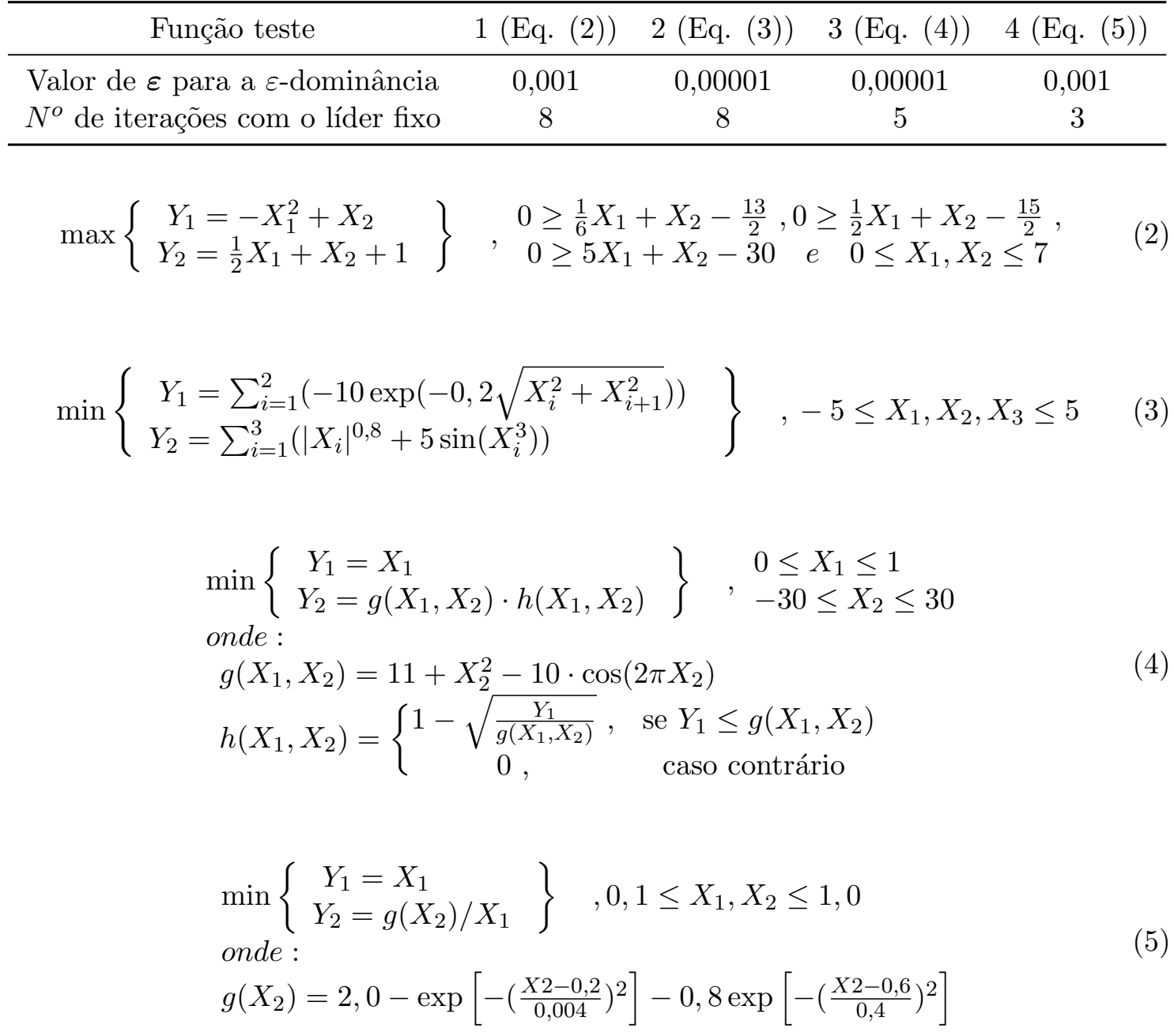

Tabela 2: Comparação numérica entre duas metodologias utilizando a métrica GD.

\begin{tabular}{lcccccc}
\hline Função & MOPSO & Melhor & Pior & Média & Mediana & Desvio padrão \\
\hline Teste 1 & Coello et al. [3] & 0,002425 & 0,476815 & 0,036535 & 0,007853 & 0,104589 \\
Eq. (2) & Proposto & 0,001011 & 0,074973 & 0,018671 & 0,011756 & 0,003509 \\
\hline Teste 2 & Coello et al. [3] & 0,00745 & 0,00960 & 0,008450 & 0,00845 & 0,00051 \\
Eq. (3) & Proposto & 0,00210 & 0,00520 & 0,003364 & 0,00319 & 0,00013 \\
\hline Teste 3 & Coello et al. [3] & 0,0000861 & 0,000191 & 0,000118 & 0,000111 & 0,0000255 \\
Eq. (4) & Proposto & 0,0000055 & 0,000392 & 0,000090 & 0,000058 & 0,0000153 \\
\hline Teste 4 & Coello et al. [3] & 0,00043 & 0,18531 & 0,03273 & 0,00051 & 0,06062 \\
Eq. (5) & Proposto & 0,00018 & 0,75106 & 0,10734 & 0,00131 & 0,02902 \\
\hline
\end{tabular}


(a)

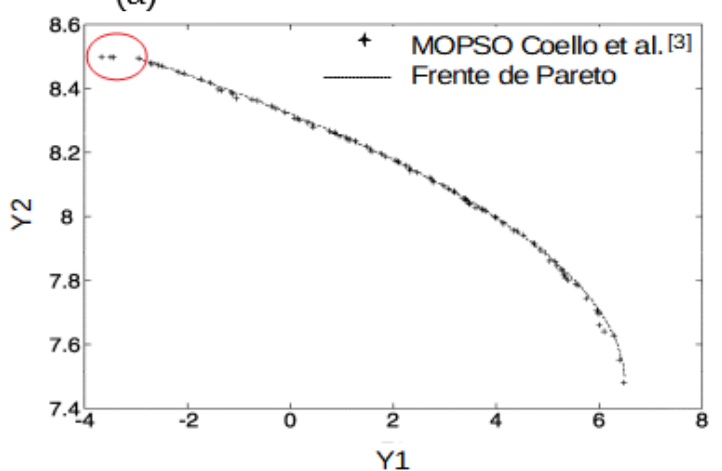

(c)

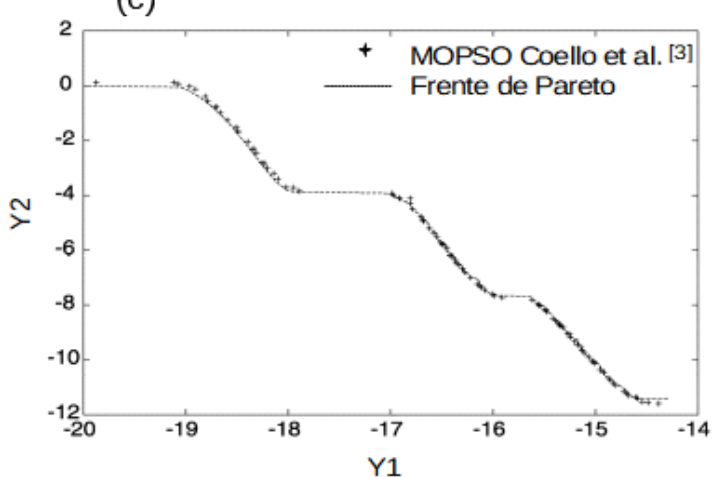

(e)

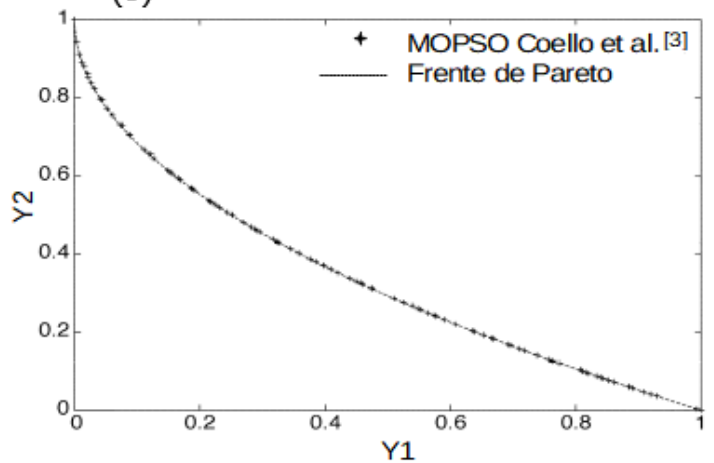

(g)

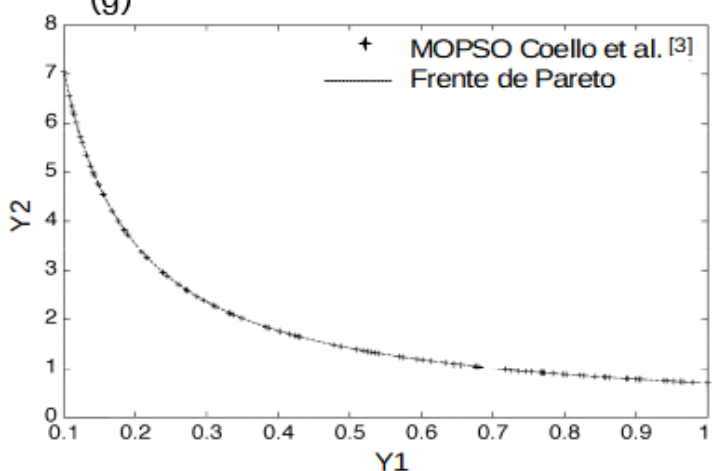

(b)

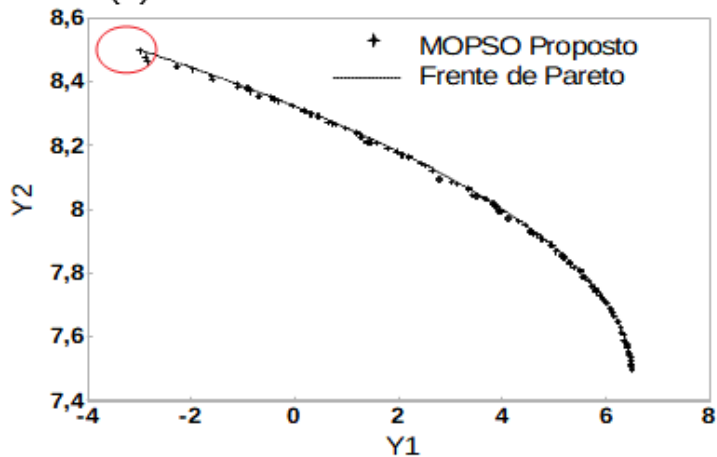

(d)
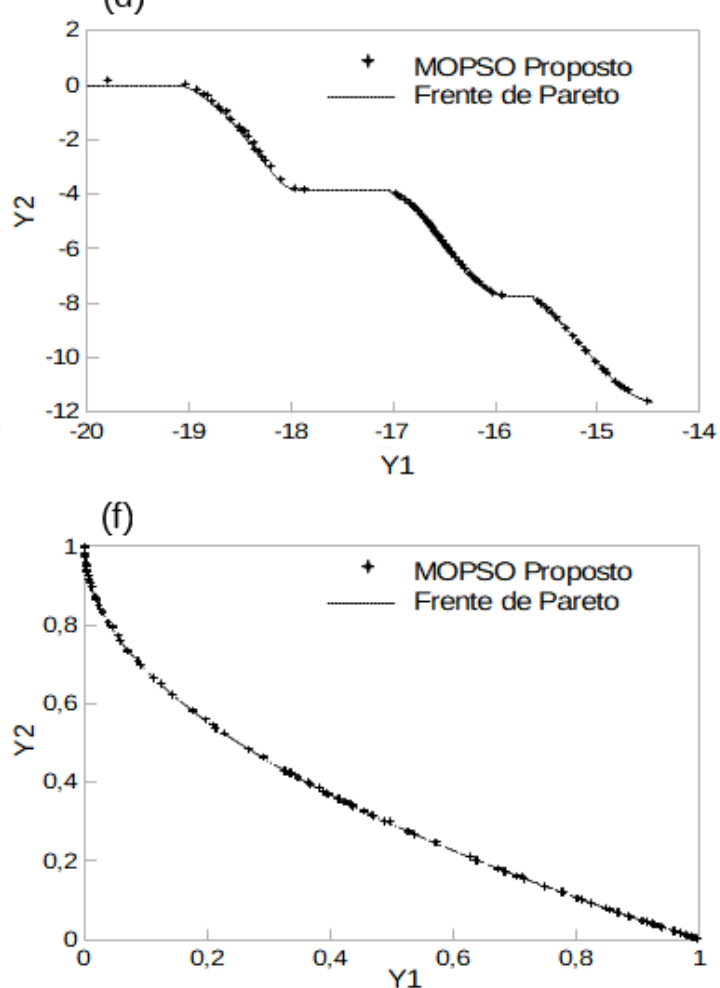

(h)

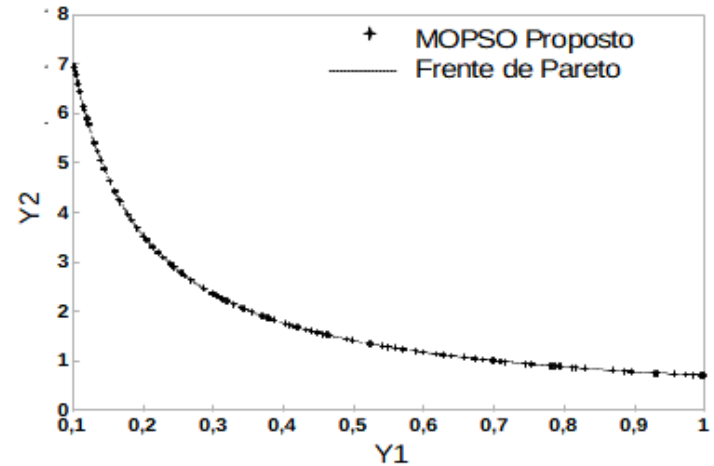

Figura 1: Comparação gráfica entre as duas metodologias para as funções teste 1 (a,b), 2 (c,d), 3 $(\mathrm{e}, \mathrm{f})$ e $4(\mathrm{~g}, \mathrm{~h})$. 
Tabela 3: Comparação numérica entre duas metodologias utilizando a métrica SP.

\begin{tabular}{lcccccc}
\hline Função & MOPSO & Melhor & Pior & Média & Mediana & Desvio Padrão \\
\hline Teste 1 & Coello et al. [3] & 0,043982 & 0,538102 & 0,109452 & 0,06748 & 0,110051 \\
Eq. (2) & Proposto & 0,005345 & 0,060613 & 0,014590 & 0,001044 & 0,001974 \\
\hline Teste 2 & Coello et al. [3] & 0,043982 & 0,538102 & 0,109452 & 0,06748 & 0,110051 \\
Eq. (3) & Proposto & 0,008786 & 0,019146 & 0,014281 & 0,014115 & 0,000383 \\
\hline Teste 3 & Coello et al. [3] & 0,00727 & 0,018676 & 0,010392 & 0,009542 & 0,002782 \\
Eq. (4) & Proposto & 0,00004 & 0,002606 & 0,000742 & 0,000339 & 0,000131 \\
\hline Teste 4 & Coello et al. [3] & 0,04007 & 0,58185 & 0,08358 & 0,05494 & 0,11821 \\
Eq. (5) & Proposto & 0,00330 & 0,67488 & 0,04463 & 0,00590 & 0,00568 \\
\hline
\end{tabular}

De forma geral, as performances das duas versões podem ser consideradas equivalentes para as quatro funções estudadas. Embora as métricas tenham apresentado resultados melhores com o algoritmo proposto (Tabelas 1 e 2), observa-se por meio da Figura 1 que ambas apresentaram uma boa distribuição de partículas e proximidade com a frente de Pareto ao longo de toda a sua extensão. Na versão proposta neste trabalho, a distribuição homogênea resulta essencialmente da eliminação das partículas com menor distância euclidiana em contrapartida à escolha sequencial dos líderes.

Observou-se que a fixação do líder por 8 iterações acelera a convergência em direção à frente de pareto, sendo adequada para problemas como os apresentados em Eqs. (2) e (3). Por outro lado, a fixação do líder por apenas 3 iterações evita uma convergência prematura, desejável para problemas como o apresentado em Eq. (5), contendo muitos mínimos locais. Verificou-se ainda que o fator de inércia e o critério de escolha da liderança local favorecem a convergência em direção à frente de Pareto.

Na Figura 1-a oberva-se a presença de alguns pontos fora da região da frente de pareto, destacados no círculo vermelho. Embora sejam não dominados, os pontos não oferecem um ganho real para a função teste 1, Eq. (2), que apresenta um platô horizontal. Estes pontos surgem devido ao que consideramos uma dominância puramente numérica. Este efeito foi reduzido pela utilização do critério de e-dominância conforme observa-se na Figura 1-b.

\section{Conclusões}

Neste trabalho foi apresentada uma nova versão do MOPSO que utiliza a estrutura original do PSO adaptada para problemas multiobjetivo a partir da implementação de seis modificações: utilização do critério de $\varepsilon$-dominância, armazenagem da liderança global (partículas não dominadas) num vetor de tamanho fixo, gerenciamento do vetor de armazenagem por meio do critério de menor distância euclidiana entre as partículas, escolha sequencial do líder global a cada grupo de 3 a 8 iterações, utilização do peso de inércia no cálculo da velocidade e utilização de um critério para diferenciação entre partículas não dominadas na escolha da liderança local.

O algoritmo proposto, além de mais simples gerou resultados ligeiramente superiores à 
versão de Coello e Lechuga [2004]. Devido à sua simplicidade esta versão apresenta grande potencial para aplicação na resolução de problemas MO, especialmente porque mantem um reduzido número de parâmetros para ajuste e efetua um gerenciamento simplificado das partículas armazenadas, sem a necessidade de cálculos e sub-rotinas complexas.

\section{Agradecimentos}

Ao Programa de Pós-doutorado da UENF (EDITAL PROPPG No 02/2018) pela bolsa do primeiro autor e à Coordenação de Aperfeiçoamento de Pessoal de Nível Superior (CAPES-Código de Financiamento 001) pela bolsa de doutorado do segundo autor.

\section{Referências}

[1] A. Chatterjee and P. Siarry. Nonlinear inertia weight variation for dynamic adaptation in particle swarm optimization. Computers $\& 3$ operations research, 33:859-871, 2006. DOI:10.1016/j.cor.2004.08.012.

[2] C. C. Coello and M. S. Lechuga. MOPSO: A proposal for multiple objective particle swarm optimization. In Proceedings of the 2002 Congress on Evolutionary Computation, 2:1051-1056, 2002. DOI: 10.1109/CEC.2002.1004388.

[3] C. A. C. Coello, G. T. Pulido and M. S. Lechuga. Handling multiple objectives with particle swarm optimization. IEEE Transactions on evolutionary computation, 8:256279, 2004. DOI:10.1109/TEVC.2004.826067.

[4] R. Eberhart and J. Kennedy. A new optimizer using particle swarm theory. In Proceedings of the Sixth International Symposium on Micro Machine and Human Science, 39-43, 1995. DOI: 10.1109/MHS.1995.494215.

[5] J. D. Knowles and D. W. Corne. Approximating the nondominated front using the Pareto archived evolution strategy. Evolutionary computation, 8:149-172, 2000. DOI: 10.1162/106365600568167.

[6] M. Laumanns, L. Thiele, K. Deb and E. Zitzler. Combining convergence and diversity in evolutionary multiobjective optimization. Evolutionary computation, 10:263-282, 2002. DOI:10.1162/106365602760234108.

[7] M. Reyes-Sierra and C. C. Coello. Multi-objective particle swarm optimizers: A survey of the state-of-the-art. International journal of computational intelligence research, 2:287-308, 2006. ISSN: 0973-1873.

[8] K. Tagawa. and N. Kojima. Multi-objective optimum design of DMS filters using robust engineering and genetic algorithm. In 2006 IEEE International Conference on Evolutionary Computation, 2208-2214, 2006. DOI: 10.1109/CEC.2006.1688580.

[9] E. Zitzler. Evolutionary algorithms for multiobjective optimization. Evolutionary Methods for Design, Optimisation, and Control, CIMNE, 19-26, 2002. (to appear) 